AIRWAY BIOLOGY

\title{
Increased matrix metalloproteinase-9 concentration and activity after stimulation with interleukin-17 in mouse airways
}

\author{
O Prause, S Bozinovski, G P Anderson, A Lindén
}

Thorax 2004;59:313-317. doi: 10.1136/thx.2003.008854

See end of article for authors' affiliations

.....................

Correspondence to: Dr O Prause, Lung Pharmacology Group, Department of Respiratory Medicine and Allergology, Institute of Internal Medicine, Göteborg University, 41346 Gothenburg, Sweden; olof.prause@ lungall.gu.se

Received 16 April 2003 Accepted

12 November 2003

\begin{abstract}
Background: The proteolytic enzyme matrix metalloproteinase (MMP)-9 can degrade structural compounds such as the extracellular matrix and the basement membrane in the airways and lungs. MMP-9 has therefore been implicated in remodelling of the airways and lungs during severe asthma and chronic obstructive pulmonary disease (COPD).

Methods: The effect of the T lymphocyte derived proinflammatory cytokine interleukin (IL)-17 on MMP-9 protein release and activity in the airways was studied in vivo and in vitro.

Results: In vivo, intranasal stimulation of mice with IL-17 induced the release of the precursor molecule proMMP-9 in bronchoalveolar lavage (BAL) fluid, associated with a pronounced local accumulation of neutrophils that stained positive for MMP-9. Stimulation with IL-17 also increased the concentration of free soluble MMP-9 that was proteolytically active as determined by a gelatinase substrate assay. The concentration of MMP-9 in BAL fluid had a strong positive correlation with the number of neutrophils; the amount of MMP-9 per neutrophil was not increased by IL-17 stimulation. In vitro, stimulation of mouse neutrophils with IL-17 did not increase the concentration of proMMP-9 in the conditioned medium.

Conclusion: Local stimulation with IL-17 increases the concentration of biologically active MMP-9 as well as its precursor molecule in mouse airways in vivo. This increase in proteolytic load is probably mainly due to an increased number of neutrophils and not to an increase in the release of MMP-9 from each neutrophil. These findings indicate a link between the T lymphocyte cytokine IL-17 and increased proteolytic load in the airways which may be relevant for chronic inflammatory airway diseases such as severe asthma and COPD.
\end{abstract}

M atrix metalloproteinases (MMPs) constitute a family of neutral proteinases that play a key role in the degradation of extracellular matrix and basement membrane in human airways and lungs. ${ }^{12}$ MMP-9 (gelatinase B, $92 \mathrm{kDa}$ type IV collagenase) is one member of the MMP family that is released as a proenzyme and subsequently activated via a protease cascade in vivo. ${ }^{3}$ The concentration of MMP-9 is increased in diseases like asthma, interstitial pulmonary fibrosis (IPF), adult respiratory distress syndrome (ARDS), and in chronic obstructive pulmonary disease (COPD). ${ }^{4-8}$ Because of its proteolytic ability, MMP-9 has been implicated in tissue remodelling of the airways and lungs in chronic inflammatory diseases such as severe asthma and COPD. ${ }^{9}$ MMP-9 is also likely to be physiologically important because of its ability to regulate the digestion of components of the extracellular matrix as well as the activity of other proteases and cytokines. ${ }^{10}$

Airway neutrophils are believed to constitute an important source of MMP-9, ${ }^{11}{ }^{12}$ even though bronchial epithelial cells, ${ }^{13}$ endothelial cells, ${ }^{14}$ and macrophages ${ }^{15}$ can also release MMP9 under certain conditions. Like the concentration of MMP-9, neutrophil numbers are increased in acute severe asthma, COPD, cystic fibrosis, and ARDS. It is currently believed that neutrophils play a pathogenetic role in these diseases ${ }^{16-20}$ by the release of various tissue degrading proteases and reactive oxygen-free radicals.

Recent studies on rodents indicate that the T lymphocyte derived cytokine interleukin (IL)-17 causes an accumulation of neutrophils in the airways in part via the release of CXC chemokines..$^{21}$ Intratracheal stimulation with IL-17 also increases the concentration of the neutrophil-derived enzymes neutrophil elastase and myeloperoxidase in rat airways in vivo. ${ }^{22}$ Interestingly, the concentration of IL-17 may also be increased in the airways of patients with acute severe asthma ${ }^{23}$ and in healthy volunteers with severe airway inflammation induced by exposure to organic dust. ${ }^{25}$ In addition, blockade of endogenous IL-17 also inhibits endotoxin induced accumulation of neutrophils in rodent airways in vivo. ${ }^{26}$ Thus, it has been proposed that IL-17 plays a central role in mobilising neutrophils in the airways and lungs. ${ }^{27}$

The aim of this study was to determine whether stimulation with IL-17 increases the load of MMP-9 in the airways and, if so, how this correlates with the accumulation of neutrophils. We first evaluated the effect of IL-17 stimulation on proMMP-9 release in mouse bronchial alveolar lavage (BAL) fluid in vivo and then determined the concentration of total soluble MMP-9 protein and the proteolytic activity against gelatine in BAL fluid. We also analysed the correlation between the neutrophil number and MMP-9 in BAL fluid and used immunocytochemistry to target BAL cells with MMP-9 protein. Finally, we studied the effect of IL-17 on the release of proMMP-9 protein from mouse neutrophils in vitro.

\section{METHODS}

\section{Animals}

This study was approved by the Animal Ethics Committee in Göteborg, Sweden (DNo. 298/2001). Pathogen-free mice (C57BL/6, male, 6-8 weeks old, B\&K Universal AB, Stockholm, Sweden) were kept in individually ventilated racks and received standard laboratory food plus water ad

Abbreviations: IL-17, interleukin 17; MMP-9, matrix metalloproteinase 9 
libidum at the Animal Care Facility of Göteborg University, Sweden.

\section{Anaesthesia and euthanasia}

Before intranasal stimulation with IL-17, the mice were transiently anaesthetised using aerosolised isofluoran (Apoteksbolaget, Göteborg, Sweden). An adequate supply of oxygen was assured. The anaesthesia did not display any long term effect on the animals after they recovered. By the end of the experiments and before collection of bronchoalveolar lavage (BAL) fluid, the animals were anaesthetised intraperitoneally using a mixture of xylazin $(130 \mathrm{mg} / \mathrm{kg}$ in $0.1 \mathrm{ml}$ sterile phosphate buffered saline, PBS) and ketamine $(670 \mathrm{mg} / \mathrm{kg}$ in $0.1 \mathrm{ml}$ PBS) (Apoteksbolaget, Göteborg, Sweden). After reaching deep anaesthesia, the mice were killed by opening the chest and bleeding the right ventricle of the heart.

\section{Local stimulation with IL-17}

Either endotoxin (LPS (lipopolysaccharide), $0.1 \mu \mathrm{g}$ in $50 \mu \mathrm{l}$ PBS; E coli serotype 026:B6, Difco Laboratories, Detroit, MI, USA), IL-17 ( $3 \mu \mathrm{g}$ in $50 \mu \mathrm{l} \mathrm{PBS}$; R\&D, Abingdon, UK) or vehicle $(50 \mu \mathrm{l}$ PBS) was administered intranasally using a micropipette. The effect of IL-17 was protein specific as shown previously when treatment with an anti-IL-17 antibody was able to inhibit IL-17 induced airway neutrophilia. ${ }^{21}$

\section{Harvest of BAL fluid and cytospin preparations}

Tracheotomy was performed and the airways were washed twice with PBS $(250 \mu \mathrm{l})$ using a tracheal cannula followed by gentle aspiration. The recovered BAL suspension was pooled and kept on ice until centrifugation (at $1000 \mathrm{r} / \mathrm{min}(70 \mathrm{~g}$ ), $10 \mathrm{~min}, 4^{\circ} \mathrm{C}$; using model 5403, Eppendorf-Netheler, Hamburg, Germany). The total number (that is, concentration) of cells was determined using resuspended cell pellets from BAL suspension. The cell-free BAL fluid supernatant was frozen for later analysis $\left(-84^{\circ} \mathrm{C}\right)$. Differential cell counts were performed on cytospin preparations (Cytospin 3, Shandon Life Sciences, Astmor, UK) after May-Giemsa staining according to standard morphological criteria using oil immersion microscopy (original magnification $\times 1000$ ). Cell counts were carried out on 400 cells and the absolute number of each cell type was calculated.

\section{Isolation and culture of neutrophils from mice}

After harvesting BAL fluid, one femur was removed and both ends were cut open. The bone marrow cells were removed by perfusion of the femur with $2.5 \mathrm{ml}$ PBS and the suspension was kept on ice until further processing.

Bone marrow cells were filtrated through a $70 \mu \mathrm{m}$ cell strainer and washed twice with PBS containing $0.5 \%$ bovine serum albumin (Sigma-Aldrich, St Louis, Missouri, USA). Neutrophils were separated using high gradient magnetic cell separation. Bone marrow cells were incubated with a monoclonal biotinylated rat anti-mouse Ly-6G (GR-1) antibody (clone RB6-8C5; PharMingen, San Diego, USA) followed by incubation with streptavidin coupled microbeads (Miltenyi Biotec, Bergisch-Gladbach, Germany). The bone marrow cells were then passed through a column (LS MACS, Miltenyi Biotec) in a magnetic field. Neutrophils were magnetically bound to the column and eluated after removal of the magnetic field. This procedure resulted in $91 \%$ purity of neutrophils in the retrieval.

Finally, the neutrophils were put in culture and stimulated with IL-17 (100 ng/ml) or endotoxin (LPS, E coli serotype 026:B6, $1 \mu \mathrm{g} / \mathrm{ml})$. RPMI medium was used as a negative control and calcium ionophore A23487 (CI: l $\mu \mathrm{M}$ ) plus phorbol-12-myristate-13-acetate (PMA: $2 \mathrm{ng} / \mathrm{ml}$ ) in RPMI was used as a positive control (both from Sigma-Aldrich).
After incubation for 24 hours, the conditioned media were harvested, centrifuged (4000 rpm ( $1000 \mathrm{~g})$ for 10 minutes), and frozen $\left(-84^{\circ} \mathrm{C}\right)$ for later analysis of mouse proMMP-9 and MMP-9.

\section{Concentration of proMMP-9}

The proMMP-9 protein concentration in BAL fluid supernatant was assessed using a commercially available mouse proMMP-9 ELISA kit (R\&D, Abingdon, UK) according to the manufacturer's instructions. This ELISA is specific for the proform of MMP-9 and can also detect proMMP-9 when complexed with tissue inhibitors of metalloproteinases (TIMPs). There is no cross reactivity between the proform and active MMP-9 according to the manufacturer.

\section{Protease expression and activity in BAL fluid}

Zymography was used to assess protease expression in response to IL-17 and LPS treatment as described previously. ${ }^{28}$ Briefly, SDS-PAGE mini-gels (10\%) were prepared with the incorporation of gelatine $(2 \mathrm{mg} / \mathrm{ml}$, Labchem) before casting. BAL fluid $(20 \mu \mathrm{l})$ was run into the gels at a constant voltage of $200 \mathrm{~V}$ under non-reducing conditions. When the dye front reached the bottom, the gels were removed and washed twice for 15 minutes in $2.5 \%$ Triton X100 and incubated at $37^{\circ} \mathrm{C}$ overnight in zymography buffer (50 mM Tris- $\mathrm{HCl}$ (pH 7.5), $5 \mathrm{mM} \mathrm{CaCl}_{2}, 1 \mathrm{mM} \mathrm{ZnCl}_{2}$ and $0.01 \% \mathrm{NaN}_{3}$ ). The gels were then stained for 45 minutes with Coomassie Blue background. BAL fluid was also tested for net gelatinase activity using fluorescence conjugated gelatin (Molecular Probes). The gelatin substrate $(10 \mu \mathrm{g})$ was diluted in $50 \mathrm{mM}$ Tris ( $\mathrm{pH} 7.5$ ), $150 \mathrm{mM} \mathrm{NaCl}, 5 \mathrm{mM} \mathrm{CaCl}_{2}, 0.01 \%$ $\mathrm{NaN}_{3}$ and incubated at room temperature for 16 hours with $100 \mu \mathrm{l}$ neat BAL fluid. The digested substrate has absorption/ emission maxima at $495 \mathrm{~nm} / 515 \mathrm{~nm}$ and its fluorescence intensity was measured in a microplate reader (Victor II, Wallac) to detect quantitative differences in activity.

\section{Detection of MMP-9 protein with immunocytochemistry (ICC)}

BAL cells from IL- 17 treated ( $3 \mu \mathrm{g} / \mathrm{animal})$ and untreated mice were fixed immediately after lavage in $4 \%$ paraformaldehyde for 20 minutes on ice. Cells were then washed with PBS twice before cytospins were performed and slides were frozen at $-84^{\circ} \mathrm{C}$

To block unspecific binding, cells were incubated in $10 \%$ donkey serum (DAKO Corp, Glostrup, Denmark) for 30 minutes. Slides were incubated with a goat anti-mouse MMP-9 antibody (1/100 from R\&D systems) or a control antibody (BD Biosciences, Oxford, UK) for 1 hour. This polyclonal MMP-9 antibody recognises both the active and the latent form of mouse MMP-9 protein. Slides were washed in Tris buffered saline (TBS) and then sequentially incubated with a biotinylated donkey anti-goat IgG (Jackson ImmunoResearch Laboratories, West Grove, PA, USA) at a dilution of 1/500, followed by alkaline-phosphatase conjugated streptavidin (DAKO Corp). After washing with TBS, antibodies were visualised using Vector Red staining (Vector Labs, Burlingame CA, USA) for 17 minutes and counterstained with Mayer's haematoxylin (DAKO Corp). Subsequently, the slides were washed in tap water, dehydrated, and mounted. Slides were evaluated using light microscopy at $1000 \times$ magnification (Zeiss, Germany) .

\section{Data analysis}

All descriptive data are presented as mean (SE) unless otherwise stated. Wilcoxon's signed rank test was used for statistical analysis of differences between groups for single paired comparisons and the Mann-Whitney U test for single 
unpaired comparisons. The Kruskall-Wallis test preceded the Mann-Whitney U test for multiple comparisons.

\section{RESULTS}

\section{ProMMP-9 in BAL fluid after stimulation with IL-17 in} vivo

IL-17 significantly increased the concentration of proMMP-9 protein in mouse BAL fluid harvested 24 hours after intranasal treatment (fig 1). Compared with the negative control, the concentration of proMMP-9 protein was increased by IL-17 stimulation by more than $1200 \%$ $(\mathrm{p}=0.03 ; \mathrm{n}=6-7)$. The proMMP-9 concentration after stimulation with IL-17 corresponded to $62(28) \%$ of the positive control (LPS). The number of neutrophils in BAL fluid was markedly increased by IL-17 stimulation, but there was no substantial effect on the number of any other cells. The ratio between the number of neutrophils and the concentration of proMMP-9 was 1.09 (0.13) for the IL-17 group and $1.39(0.41)$ for the negative control group.

Release of MMP-9 after stimulation with IL- 17 in vivo Stimulation with IL-17 markedly increased the concentration of active and latent MMP-9 protein in mouse BAL fluid harvested 24 hours after intranasal treatment with IL-17 (fig. 2). More than $90 \%$ of the gelatinase activity present on zymography corresponded to MMP-9 (data not shown); no substantial concentrations of any other MMPs were detectable. The concentration of MMP-9 protein was increased by more than $800 \%$ compared with the negative control (PBS) and constituted $59(20) \%$ of the positive control (LPS).

\section{Net protease activity as measured by gelatinase substrate assay}

Intranasal stimulation with IL-17 caused a 251 (25)\% increase in net gelatinase activity in mouse BAL fluid 24 hours after treatment compared with the negative control (fig 3). The IL-17 induced increase in gelatinase activity corresponded to $60(20) \%$ of the gelatinase activity induced by stimulation with LPS (positive control).

\section{Correlation between concentration of MMP-9 and number of neutrophils in BAL fluid}

Stimulation with IL-17 caused an increase of more than $900 \%$ in the number of neutrophils in mouse BAL fluid harvested 24 hours later. After stimulation with IL-17 the concentration of MMP-9 protein (fig 4A) correlated strongly with the number of neutrophils in BAL fluid (Spearman rank correlation: rho $=0.9, \mathrm{p}=0.04)$. There was no statistically

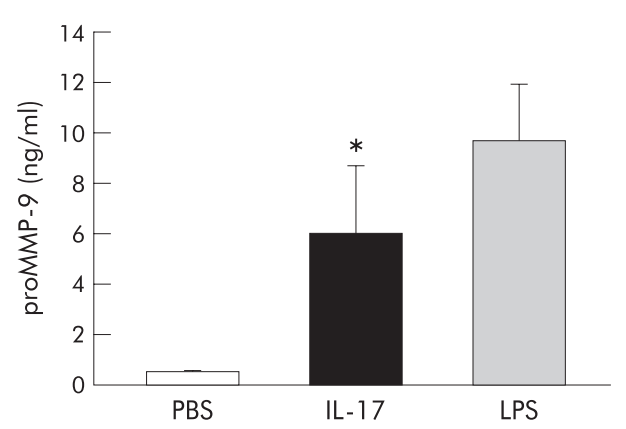

Figure 1 Concentration of proMMP-9 $(\mathrm{ng} / \mathrm{ml}$ ) in bronchoalveolar lavage (BAL) fluid from mice harvested 24 hours after intranasal treatment either with interleukin (IL)-17 (3 $\mathrm{\mu g} /$ animal), LPS $(0.1 \mu \mathrm{g} /$ animal), or PBS (negative control) as determined by ELISA. Data presented as mean (SE); $n=6-7 .{ }^{*} p=0.03$ for treatment with IL-17 compared with negative control (Mann-Whitney $U$ test).
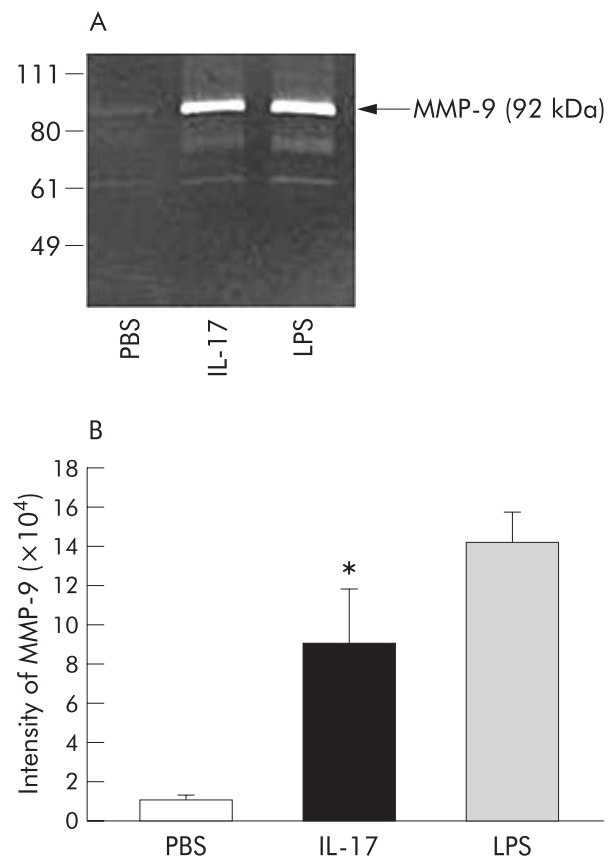

Figure 2 (A) Gelatin zymography of bronchoalveolar lavage (BAL) fluid from mice 24 hours after intranasal treatment either with PBS (negative control), interleukin (IL)-17 (3 $\mu \mathrm{g} /$ animal), or LPS $(0.1 \mu \mathrm{g} /$ animal) showing the proteolytic activity present on a zymography gel slab. A major band of proteolytic activity was observed between 111 and $80 \mathrm{kDa}$ which corresponds to the molecular weight of MMP-9 $(92 \mathrm{kDa})$. This band was then subjected to densitometry using Kodak 1D image analysis software and presented graphically (B). Data presented as mean (SE); $n=6-7$. ${ }^{*} p=0.04$ for treatment with IL-17 compared with negative control (Mann-Whitney $U$ test).

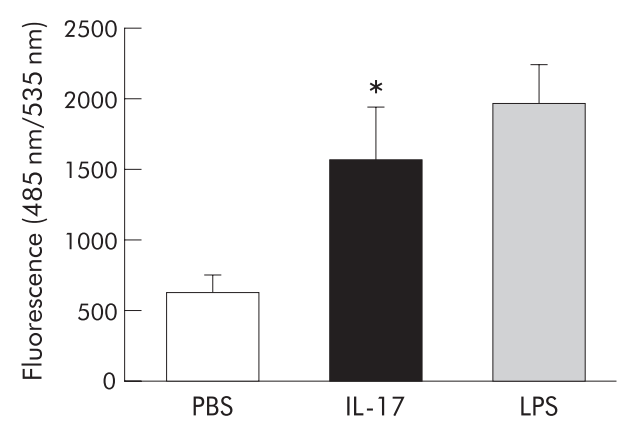

Figure 3 Net gelatinase activity determined using a gelatin conjugated fluorescence substrate assay in BAL fluid from mice 24 hours after intranasal treatment with IL-17 (3 $\mathrm{gg} /$ animal), LPS $(0.1 \mu \mathrm{g} /$ animal $)$, or PBS (negative control). Data presented as mean (SE); $n=6-7 .{ }^{*} p=0.02$ for treatment with IL-17 compared with negative control (Mann-Whitney $U$ test).

significant correlation between the concentration of MMP-9 and the number of macrophages in BAL fluid (fig 4B; Spearman rank correlation: rho $=-0.3, \mathrm{p}=0.56$ )

\section{Detection of MMP-9 protein by ICC}

ICC staining with a specific detection anti-mouse MMP-9 antibody was positive for neutrophils (fig 5B and 5C) but not for any other cell type including airway macrophages (fig 5A) in BAL fluid from mice stimulated intranasally with mouse IL-17. BAL fluid from naive animals stained positive only for neutrophils as well (data not shown). No non-specific staining was detected using the isotype control antibody. 

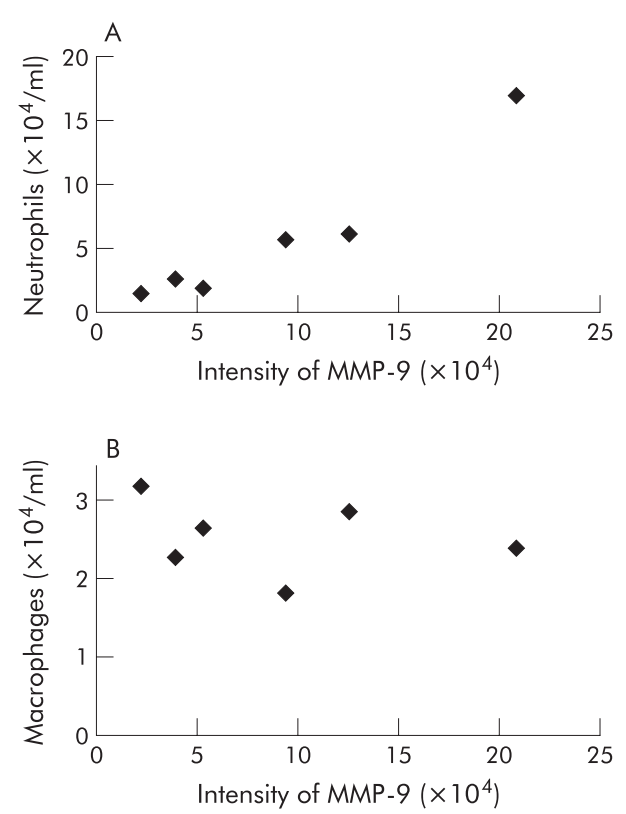

Figure 4 Correlation between number of (A) neutrophils and (B) macrophages in BAL fluid from mice 24 hours after intranasal treatment with IL-17 (3 $\mathrm{gg} /$ animal) and the intensity of MMP-9 release in BAL fluid determined by measuring the net intensity of the corresponding band $(92 \mathrm{kDa})$. Spearman rank correlation: $(A)$ rho $=0.942, p=0.04$; $(B)$ rho $=-0.257, p=0.56 ; n=6-7)$.

\section{Release of proMMP-9 from neutrophils in vitro}

Stimulation of isolated mouse neutrophils with IL-17 during a 24 hour period did not increase the concentration of proMMP-9 protein in the conditioned medium of cultured cells compared with the negative control (PBS). However, stimulation with LPS and the positive control (CI/PMA), respectively, increased the concentration of proMMP-9 protein in the conditioned medium harvested from isolated neutrophils 24 hours later (fig 6).

\section{DISCUSSION}

This study shows that intranasal stimulation with the $\mathrm{T}$ lymphocyte cytokine IL-17 causes a pronounced accumulation of neutrophils in mouse BAL fluid in vivo that is associated with an increase in the local proMMP-9 concentration. The study also shows that stimulation with IL-17 increases the concentration of active free and soluble MMP-9 and that more than $90 \%$ of the local gelatinase activity corresponds to MMP-9. In vitro, IL-17 does not directly stimulate the release of proMMP-9 from neutrophils. Taken together, the results indicate that IL-17 causes an increased proteolytic load dominated by MMP-9 in the airways in vivo and that neutrophils constitute the most prominent source of this MMP-9.

We propose that the neutrophil is a source of MMP-9 in mouse airways after local stimulation with IL-17 for several reasons. Firstly, the number of neutrophils correlates strongly with the concentration of MMP-9 in BAL fluid and the same is true for proMMP-9 (data not shown). Secondly, we found a substantial inherent release of proMMP-9 from purified neutrophils in vitro, even though the release appears to be independent of direct IL-17 stimulation. Thirdly, other investigators have shown that removal of circulating blood neutrophils by pretreatment with vinblastine results in severe suppression of MMP-9 activity in plasma from endotoxinexposed mice in vivo, again suggesting that neutrophils are an important source of MMP-9. ${ }^{29}$ Fourthly, neutrophils from BAL fluid taken from mice after stimulation with IL-17 display positive ICC staining for MMP-9 while other cell types, including macrophages, do not show any such staining.

The putative role of airway macrophages in the IL-17induced increase in MMP-9 concentration is uncertain. Firstly, we and others have recently shown that endotoxin induced accumulation of neutrophils, but not macrophages, depends upon endogenous IL-17 in the airways. ${ }^{26}{ }^{30}$ Secondly, as shown in this and other studies on Thl-like conditions, the number of macrophages in BAL fluid is not increased by IL$17 .{ }^{26}{ }^{30}$ Thirdly, in our current in vivo study the correlation between the number of macrophages and the MMP-9 concentration in the BAL fluid was weak and was not statistically significant. Fourthly, in a previous study on isolated human macrophage-like cells in vitro, the phenomenon that IL-17 caused the release of MMP-9 appeared at a much later time point than we used in our current study. ${ }^{31}$ Taken together, these facts suggest neutrophils-but do not completely rule out macrophages-as the most important source of MMP-9 after stimulation with IL-17 in the airways in vivo.

Interestingly, when we previously showed that IL-17 increases neutrophil elastase and myeloperoxidase concentrations in rat airways in vivo, we also showed that rat neutrophils do not release myeloperoxidase or neutrophil elastase after direct stimulation by IL-17 in vitro. ${ }^{22}$ Just as in the current study, the negative in vitro finding contrasts with the positive in vivo finding on IL-17. In view of previous studies, it seems very likely that this can be explained by IL17 acting mainly on the recruitment of neutrophils but not on their activation per se. This explanation is supported not only from our in vitro data, but also from our in vivo study that showed no increase in the release of MMP-9 per neutrophil in BAL fluid after stimulation with IL-17 and no difference in neutrophil staining for MMP-9 after ICC in IL-17 treated
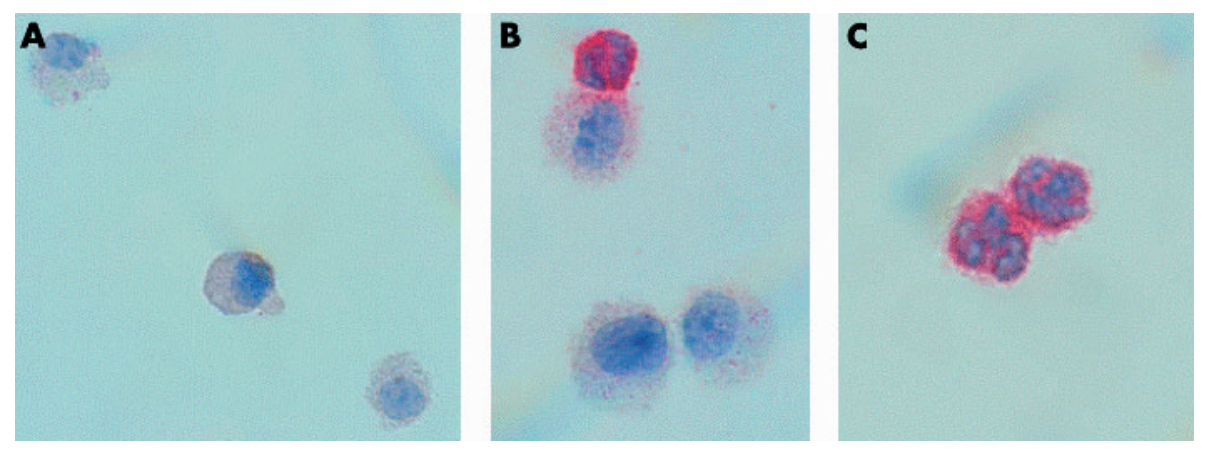

Figure 5 Immunocytochemical detection of mouse MMP-9 protein in BAL fluid cells harvested 24 hours after intranasal treatment with IL-17 ( $3 \mu \mathrm{g} /$ animal). (A) Airway macrophages alone; (B) airway macrophages and neutrophils; and (C) neutrophils alone. 


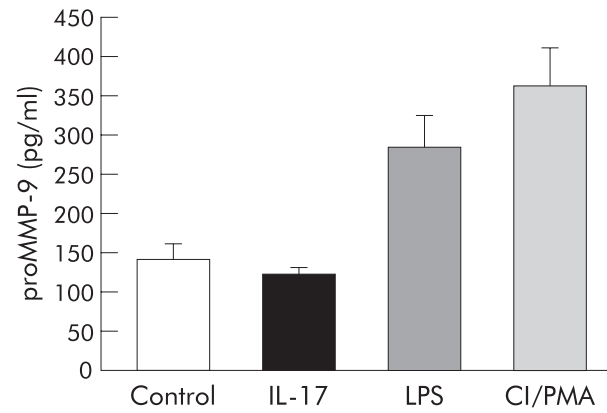

Figure 6 Release of soluble proMMP-9 protein in conditioned medium from isolated mouse neutrophils cultured for 24 hours and stimulated either with PBS (negative control), IL-17 $(100 \mathrm{ng} / \mathrm{ml})$, LPS $(1 \mu \mathrm{g} / \mathrm{ml})$, or $\mathrm{Cl} / \mathrm{PMA}$ ( $1 \mu \mathrm{M}$ and $2 \mathrm{ng} / \mathrm{ml}$ respectively; positive control) in vitro. Data presented as mean (SE); $n=4$.

animals compared with naïve animals. In the context of neutrophil recruitment, MMP-9 may therefore orchestrate the migration of neutrophils through the airway wall by degrading components of the vascular endothelial-cadherin complex during neutrophil-endothelial contact. ${ }^{32}{ }^{33}$ It has therefore been discussed whether MMP-9 constitutes an important factor for the recruitment of neutrophils in previous studies. ${ }^{34} 35$ However, the mechanistic role of MMP-9 in neutrophil recruitment after stimulation with IL17 needs to be further investigated.

In conclusion, this study shows that local stimulation with the T lymphocyte cytokine IL-17 increases the concentration of MMP-9 protein and the net proteolytic activity in association with a pronounced accumulation of neutrophils in mouse airways in vivo. It appears likely that the increase in proteolytic activity is caused by an accumulation of neutrophils and not by their activation. Other studies on mice suggest that IL-17 and MMP-9 are important endogenous factors in host defence in mice. ${ }^{36}{ }^{37}$ If similar mechanisms exist in human airways, then T lymphocytes and IL-17 may be involved in controlling MMP-9 associated defence mechanisms in the airways and in the lungs. This means that IL-17 constitutes a potential pharmacotherapeutic target for the treatment of airway and lung diseases characterised by neutrophil accumulation and tissue degradation.

\section{Authors' affiliations}

O Prause, A Lindén, Lung Pharmacology Group, Department of Respiratory Medicine and Allergology, Institute of Internal Medicine, Göteborg University, Gothenburg, Sweden

S Bozinovski, G P Anderson, Lung Disease Research Laboratories, CRC for Chronic Inflammatory Diseases, Department of Pharmacology, the University of Melbourne, Parkville, VIC 3010, Australia

\section{REFERENCES}

1 Birkedal-Hansen H, Moore WG, Bodden MK, et al. Matrix metalloproteinases: a review. Crit Rev Oral Biol Med 1993:4:197-250.

2 Atkinson JJ, Senior RM. Matrix metalloproteinase-9 in lung remodeling. Am J Respir Cell Mol Biol 2003;28:12-24.

3 Cuzner ML, Opdenakker G. Plasminogen activators and matrix metalloproteases, mediators of extracellular proteolysis in inflammatory demyelination of the central nervous system. J Neuroimmunol 1999;94:1-14.

4 Lee YC, Lee HB, Rhee YK, et al. The involvement of matrix metalloproteinase-9 in airway inflammation of patients with acute asthma. Clin Exp Allergy 2001;31:1623-30.

5 Suga $M$, lyonaga $K$, Okamoto $T$, et al. Characteristic elevation of matrix metalloproteinase activity in idiopathic interstitial pneumonias. Am J Respir Crit Care Med 2000;162:1949-56.

6 Torii K, lida K, Miyazaki Y, et al. Higher concentrations of matrix metalloproteinases in bronchoalveolar lavage fluid of patients with adult respiratory distress syndrome. Am J Respir Crit Care Med 1997;155:43-6.
7 Keatings VM, Barnes PJ. Granulocyte activation markers in induced sputum: comparison between chronic obstructive pulmonary disease, asthma, and normal subjects. Am J Respir Crit Care Med 1997; 155:449-53.

8 Betsuyaku T, Nishimura M, Takeyabu K, et al. Neutrophil granule proteins in bronchoalveolar lavage fluid from subjects with subclinical emphysema. Am J Respir Crit Care Med 1999;159:1985-91.

9 Ohnishi K, Takagi M, Kurokawa Y, et al. Matrix metalloproteinase-mediated extracellular matrix protein degradation in human pulmonary emphysema. Lab Invest 1998;78:1077-87.

10 Shapiro SD, Senior RM. Matrix metalloproteinases. Matrix degradation and more. Am J Respir Cell Mol Biol 1999;20:1100-2.

11 Zaoui P, Barro C, Morel F. Differential expression and secretion of gelatinases and tissue inhibitor of metalloproteinase-1 during neutrophil adhesion. Biochim Biophys Acta 1996;1290:101-12.

12 Campbell EJ, Senior RM, Welgus HG. Extracellular matrix injury during lung inflammation. Chest 1987;92:161-7.

13 Yao PM, Lemjabbar H, D'Ortho MP, et al. Balance between MMP-9 and TIMP1 expressed by human bronchial epithelial cells: relevance to asthma. Ann NY Acad Sci 1999;878:512-4.

14 Schnaper HW, Grant DS, Stetler-Stevenson WG, et al. Type IV collagenase(s) and TIMPs modulate endothelial cell morphogenesis in vitro. J Cell Physiol 1993;156:235-46.

15 Welgus HG, Campbell EJ, Cury JD, et al. Neutral metalloproteinases produced by human mononuclear phagocytes. Enzyme profile, regulation, and expression during cellular development. $j$ Clin Invest 1990;86:1496-502.

16 Saetta M, Turato G, Facchini FM, et al. Inflammatory cells in the bronchial glands of smokers with chronic bronchitis. Am J Respir Crit Care Med 1997; 156:1633-9.

17 Fahy JV, Kim KW, Liu J, et al. Prominent neutrophilic inflammation in sputum from subjects with asthma exacerbation. J Allergy Clin Immunol 1995;95:843-52.

18 Meyer KC, Zimmerman J. Neutrophil mediators, Pseudomonas, and pulmonary dysfunction in cystic fibrosis. J Lab Clin Med 1993;121:654-61.

19 Blackwell TS, Christman JW. Defining the lung's response to endotoxin. Am J Respir Crit Care Med 2001;163:1516-7.

20 Lindén A, Adachi M. Neutrophilic airway inflammation and IL-17. Allergy 2002; 57:769-75.

21 Laan M, Cui ZH, Hoshino $\mathrm{H}$, et al. Neutrophil recruitment by human IL-17 via C-X-C chemokine release in the airways. J Immunol 1999;162:2347-52.

22 Hoshino $H$, Laan $M$, Sjöstrand $M$, et al. Increased elastase and myeloperoxidase activity associated with neutrophil recruitment by IL-17 in airways in vivo. J Allergy Clin Immunol 2000;105:143-9.

23 Molet S, Hamid Q, Davoine F, et al. IL-17 is increased in asthmatic airways and induces human bronchial fibroblasts to produce cytokines. J Allergy Clin Immunol 2001;108:430-8.

24 Lindén A. Role of interleukin-17 and the neutrophil in asthma. Int Arch Allergy Immunol 2001;126:179-84.

25 Laan M, Palmberg L, Larsson K, et al. Free, soluble interleukin-17 protein during severe inflammation in human airways. Eur Respir J 2002; 19:534-7.

26 Miyamoto M, Prause O, Sï̈strand M, et al. Endogenous IL-17 as a mediator of neutrophil recruitment caused by endotoxin exposure in mouse airways. $\mathrm{J}$ Immunol 2003;170:5335-42.

27 Kolls JK, Kanaly ST, Ramsay AJ. Interleukin-17: an emerging role in lung inflammation. Am J Respir Cell Mol Biol 2003;28:9-11.

28 Bozinovski S, Jones JE, Vlahos R, et al. Granulocyte/macrophage-colonystimulating factor (GM-CSF) regulates lung innate immunity to lipopolysaccharide through Akt/Erk activation of NFkappa B and AP-1 in vivo. J Biol Chem 2002;277:42808-14.

29 Aoki K, Ishida Y, Kikuta N, et al. Role of CXC chemokines in the enhancement of LPS-induced neutrophil accumulation in the lung of mice by dexamethasone. Biochem Biophys Res Commun 2002;294:1101-8.

30 Ferretti S, Bonneau O, Dubois GR, et al. IL-17, Produced by lymphocytes and neutrophils, is necessary for lipopolysaccharide-induced airway neutrophilia: IL-15 as a possible trigger. J Immunol 2003:170:2106-12.

31 Jovanovic DV, Martel-Pelletier J, Di Battista JA, et al. Stimulation of 92-kd gelatinase (matrix metalloproteinase 9) production by interleukin-17 in human monocyte/macrophages: a possible role in rheumatoid arthritis. Arthritis Rheum 2000;43:1134-44.

32 Allport JR, Ding H, Collins T, et al. Endothelial-dependent mechanisms regulate leukocyte transmigration: a process involving the proteasome and disruption of the vascular endothelial-cadherin complex at endothelial cell-tocell junctions. J Exp Med 1997;186:517-27.

33 Del Maschio A, Zanetti A, Corada M, et al. Polymorphonuclear leukocyte adhesion triggers the disorganization of endothelial cell-to-cell adherent junctions. J Cell Biol 1996; 135:497-510.

34 Delclaux C, Delacourt C, D'Ortho MP, et al. Role of gelatinase B and elastase in human polymorphonuclear neutrophil migration across basement membrane. Am J Respir Cell Mol Biol 1996;14:288-95.

35 Betsuyaku T, Shipley JM, Liu Z, et al. Neutrophil emigration in the lungs, peritoneum, and skin does not require gelatinase B. Am J Respir Cell Mol Biol 1999:20:1303-9.

36 Ye P, Garvey PB, Zhang P, et al. Interleukin- 17 and lung host defense against Klebsiella pneumoniae infection. Am J Respir Cell Mol Biol 2001;25:335-40.

37 Bottcher T, Spreer A, Azeh I, et al. Matrix metalloproteinase-9 deficiency impairs host defense mechanisms against Streptococcus pneumoniae in a mouse model of bacterial meningitis. Neurosci Lett 2003;338:201-4. 